ORIGINAL PAPER

\title{
Elevated expression of IGFL1 indicates UNFAVORABle PROGNOSIS IN LUNG ADENOCARCINOMA THROUGH PROMOTION OF CELL PROLIFERATION AND INHIBITION OF APOPTOSIS
}

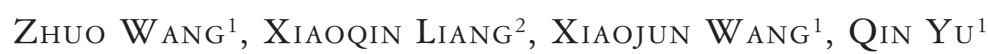

${ }^{1}$ The First Clinical Medical College of Lanzhou University, China

${ }^{2}$ Gansu Province People's Hospital, China

\begin{abstract}
Non-small cell lung cancer (NSCLC) is one of the principal causes of death worldwide. The disease is often diagnosed late, at the stage of metastasis, which leads to a poor prognosis. With currently available treatment, most patients experience relapse and drug resistance.

This study aimed to determine the potential role of insulin growth factor-like family member 1 (IGFL1) in lung adenocarcinoma (LUAD).

We analyzed the expression of IGFL1 using TIMER, UALCAN, and GEPIA. The association of IGFL1 expression with symptoms and overall survival (OS) in LUAD patients was evaluated using a tissue microarray (TMA). Moreover, A549 and H1299 cells with selective IGFL1 knockdown were used to observe their proliferation and apoptosis in vitro.

From the TIMER, UALCAN and GEPIA databases, we found that IGFL1 was upregulated in LUAD ( $\mathrm{p}<0.001)$. The level of promoter methylation in IGFL1 was significantly downregulated in LUAD and patient groups with different gender. Similar results were obtained in UALCAN. The semi-quantitative analysis revealed that IGFL1 expression is correlated with TNM stage $(\mathrm{p}<0.001)$ and tumor size $(\mathrm{p}<0.001)$ in TMA. High levels of IGFL1 expression were associated with poor OS ( $p<0.05$ ). Functional tests in A549 and H1299 cells showed that knocking down the expression of IGFL1 inhibited proliferation and enhanced apoptosis.

Our study revealed the potential utility of IGFL1 as a predictive biomarker, which may play a biological role by regulating the proliferation and apoptosis of tumor cells.
\end{abstract}

Key words: insulin growth factor-like family member 1, lung adenocarcinoma, biomarker, prognosis, proliferation, apoptosis.

\section{Introduction}

Lung cancer arises from the bronchial mucosal epithelium and is the leading cause of cancer incidence and mortality worldwide [1]. Approximately one-quarter of all cancer deaths are due to lung cancer. Non-small cell lung cancer (NSCLC) is the most common type of lung cancer, accounting for approximately $85 \%$ of all cases [2]. With conventional treatments, including surgical resection, chemotherapy, and radiation therapy, the five-year survival rate is less than $15 \%$. Lung adenocarcinoma (LUAD) is a predominant histological type of NSCLC in recent decades with an increasing amount of cases. Unfortunately, most new cases of LUAD are diagnosed at an advanced stage when the best time 
for diagnosis and treatment has already been missed. The prospects to identify the risk factors in patients with surgically resected LUAD who may have a high risk of recurrence and a poor prognosis are limited. The tumor-nodal-metastasis (TNM) system, which was revised in 2017, is the most important prognostic factor for survival [3]. In addition to TNM stage, several clinical parameters, including visceral pleural invasion [4], tumor size [5], and specific LUAD histologic subtypes [6], are also associated with poor prognosis. Along with the development of genomic analysis and immunologic profiling, variations in several genes were found to be associated with poor outcomes, including mutations in KRAS, P53, PI3K, and PD-L1 immune reactivity [7]. In recent years, with the development and application of molecular targeted therapy, understanding the molecular pathways and their alterations in LUAD has become crucial. Therefore, it is particularly important to find new biomarkers to provide the possibility of improving the prognosis of patients.

Insulin growth factor (IGF)-like family member 1 (UNQ644, APRG644), also known as IGF-like (IGFL) family member 1 (IGFL1), belongs to the IGFL family and is a novel secreted protein [8], which was discovered and reported in Homo sapiens in 2006. In humans, the four members of the IGFL family (IGFL1-4) and two pseudo-genes (IGFL1P1 and IGFL1P2) are located on chromosome 19 within a $220 \mathrm{~kb}$ interval[8]. In addition to the ovary, spinal cord, and fetal skin, IGFL1 was observed in head and neck tumors, uterine tumors, and squamous cell carcinomas $[8] \mathrm{e}>$. To date, the biological functions and gene interactions of IGFL1 are not very clear; however, the structure and sequence suggest that IGFL proteins are related to the superfamily of IGFs [8]. In many cancers, both IGFLs and IGFs share similar gene expression patterns, and play multiple roles in regulating proliferation, differentiation, and apoptosis of cancer cells. Several studies have shown that high serum concentrations of IGFs are predictive of increased cancer risk, including various types of lung cancers [9]. Previous studies detected IGFL1 expression in the skin of psoriasis patients [10]. In the study of $\mathrm{HaCaT}$ keratinocytes chronically treated with arsenic trioxide, upregulation of IGFL1 was found to be associated with metaplasia induced by chronic inflammation and ultimately carcinogenesis of skin keratinocytes [11]. Moreover, to study the effects of tumor-associated macrophages (TAMs) on the tumor microenvironment of esophageal squamous cell carcinomas (ESCCs), we confirmed that IGFL1 mRNA was downregulated in the co-culture ESCC cell lines [12]. The current findings reveal that the expression of IGFL1 is divergent in different cancers, which suggests that IGFL1 functions both as an oncogene and as a tumor suppressor, depending on the type of tumor. To date, the effect of IGFL1 on the development and pathology of LUAD has rarely been studied. In the present study, we evaluated the expression and prognosis of IGFL1 in LUAD using bioinformatic analysis. Moreover, we utilized a large number of clinical samples as well as cellular and biological data to further explore the role of IGFL1. This study aimed to investigate the expression and prognosis of IGFL1 in LUAD tissues and to understand the biological functions of IGFL1 in LUAD cells.

\section{Material and methods}

\section{Bioinformatic analysis and prediction}

\section{Tumor Immune Estimation Resource (TIMER)}

TIMER is a tool for the systematic analysis of immune infiltrates in different cancer types using The Cancer Genome Atlas (TCGA) profiling data [13]. Additionally, different expression modules were used to evaluate the differential expression of IGFL1 in tumor tissues and adjacent normal tissues in all TCGA tumors, including LUAD.

\section{University of Alabama Cancer Database (UALCAN)}

The expression levels of IGFL1 in primary LUAD were analyzed using the UALCAN online tool, which is a TCGA online analysis and mining website [14]. In the present study, we analyzed the expression of IGFL1 in LUAD, and then the promoter methylation status in LUAD, with different clinical parameters.

\section{Gene Expression Profiling Interactive Analysis (GEPIA)}

GEPIA is an integrated online database developed by scientists at Peking University, which provides data on 9736 tumors and 8587 control samples from TCGA and Genotype Tissue Expression projects [15]. We compared the mRNA expression levels of IGFL1 in LUAD samples.

\section{Kaplan-Meier plotter}

The Kaplan-Meier plotter is a practical tool with a powerful role in analyzing the relationship between gene expression and the prognosis of patients with 21 cancer types [16]. This study aimed at exploring the correlation between the mRNA expression of IGFL1 and overall survival (OS) of lung cancer and LUAD patients.

\section{Cancer Cell Line Encyclopedia (CCLE)}

CCLE is a database of cancer cell lines maintained by the Broad Institute of MIT and Harvard. It is an open-access database, which includes 10,897 sam- 
ples across 32 cancer types from TCGA to estimate the mRNA expression of cancer cells [17]. We used the CCLE database to analyze the mRNA expression levels of IGFL1 in various cancer cell lines.

\section{Patient cohort}

This research was granted scientific and ethical approval by The Ethics Committee of Gansu Provincial People's Hospital. The aim was to evaluate the correlation between the expression of IGFL1 and clinical features and prognosis in LUAD patients. The samples were harvested from 208 patients who underwent complete surgical resection for LUAD between January 2013 and December 2015 at Gansu Provincial People's Hospital. The studies were compliant with the following criteria: (1) the samples were obtained from patients with surgically resected LUAD; (2) histological type of cancer was confirmed as LUAD; and (3) relevant clinical feature data were collected from a prospectively maintained database. Moreover, (4) they were pathologic reports $\left(8^{\text {th }}\right.$ edition of AJCC Cancer Staging Manual); (5) classifying invasive adenocarcinoma according to the 2015 WHO classification [18] and the 2011 IASLC/ATS/ ERS classification [19]; and (6) providing corresponding survival data. Exclusion criteria included: (1) adenosquamous carcinoma; (2) accepted preoperative chemotherapy/radiotherapy or molecular targeted therapy; (3) metastatic LUAD; and (4) pathologic stage IV disease. The screening process for all subjects meeting the conditions is presented in Figure 4. The predominant histologic subtype was the pattern with the highest percentage. Tumors were classified as lepidic (LPA, $\mathrm{n}=47$ ), acinar (APA, $\mathrm{n}=38$ ), papillary (PPA, $\mathrm{n}=40)$, micropapillary (MPA, $\mathrm{n}=39$ ), or solid (SPA, $\mathrm{n}=44$ ) and grouped according to architectural grade: low, intermediate, and high. Para-cancerous lung tissue outside the tumor was histologically normal.

\section{Tissue microarray (TMA) construction and immunohistochemical staining}

We used formalin-fixed, paraffin-embedded cancer tissue specimens to perform the TMA. In brief, representative areas from the most predominant histologic sections were marked on hematoxylin and eosin-stained tissues, and cylindrical $0.5-\mathrm{mm}$ tissue cores were arrayed from the corresponding paraffin blocks into a recipient block using a tissue arrayer. For each block, 4-um-thick tissue sections were serially cut, transferred onto charged glass slides, and deparaffinized. Antigen retrieval was performed using a citrate buffer ( $\mathrm{pH}$ 6.0). The standard avidin-biotin-peroxidase complex was used for immunostaining of the anti-IGFL1 antibody (Invitrogen; Cat\# PA5100830; AB_2850331; dilution 1:100). Sections were stained using a Ventana Benchmark XT automated immunohistochemical stainer.

The stained sections were analyzed by two pathologists in a blind trial. The expression was initially evaluated by both staining intensity and percentage of staining-positive cells, according to a semi-quantitative scoring system. The expression of IGFL1 was observed in the cytoplasm of LUAD cells. Staining intensity was scored as 0 (negative), 1 (weak), 2 (moderate), and 3 (strong). The percentage of positive cells was quantified as 0 for $\leq 5 \%$ positive cells, 1 for $6-25 \%, 2$ for $26-50 \%, 3$ for $51-75 \%$, and 4 for $\geq 76 \%$. The final score was then generated by multiplying the score of staining intensity and percentage of positive cells (0-12). Based on the immune reactivity score of IGFL1 patients, patients were divided into two subgroups: $<6$ into a subgroup with low expression and $\geq 6$ into a subgroup with high expression [20].

\section{Lentiviral shRNA-mediated IGFL1 silencing}

The human LUAD cell lines A549, H1299, and H1975 and normal lung epithelial cells (BEAS-2B) were purchased from the Institute of Cell Biology of the Chinese Academy of Sciences. The shIGFL1 sequence was 5'-AGCTGATAAACCAGAACTG-3', and the shCtrl sequence was 5'-TTCTCCGAACGTGTCACGT-3'. Transfection was performed using the designated reagent according to the manufacturer's instructions, followed by an additional incubation for $72 \mathrm{~h}$. Transfection efficiency was confirmed based on the percentage of green fluorescent protein-positive cells under a fluorescence microscope (IX71, Olympus, Japan). Stable knockdown of IGFL1 in selected cells was confirmed via reverse transcription polymerase chain reaction (RT-PCR).

\section{RT-PCR}

The mRNA expression of IGFL1 in both A549 and H1299 cells was analyzed via RT-PCR using the LightCycler 480 II real-time PCR instrument (Roche). Briefly, total cellular RNA was extracted using TRIzol (Thermo Fisher Scientific). The RNA was then quantified using a Nanodrop spectrophotometer 2000/2000C (Thermo Fisher Scientific), and reverse transcribed into cDNA using the M-MLV kit (Promega), according to the manufacturer's instructions. Amplification was performed using the SYBR Master Mix (Takara). Each procedure was repeated three times. The relative expression level of IGFL1 was calculated using the $2^{-\Delta \Delta c t}$ method and normalized by comparing it to the housekeeping gene GAPDH. The primers used are listed in Supplementary Table S1.

\section{Cell proliferation assay}

The Cell Counting Kit-8 (CCK-8) assay was used to measure cell proliferation. Briefly, different groups 
A

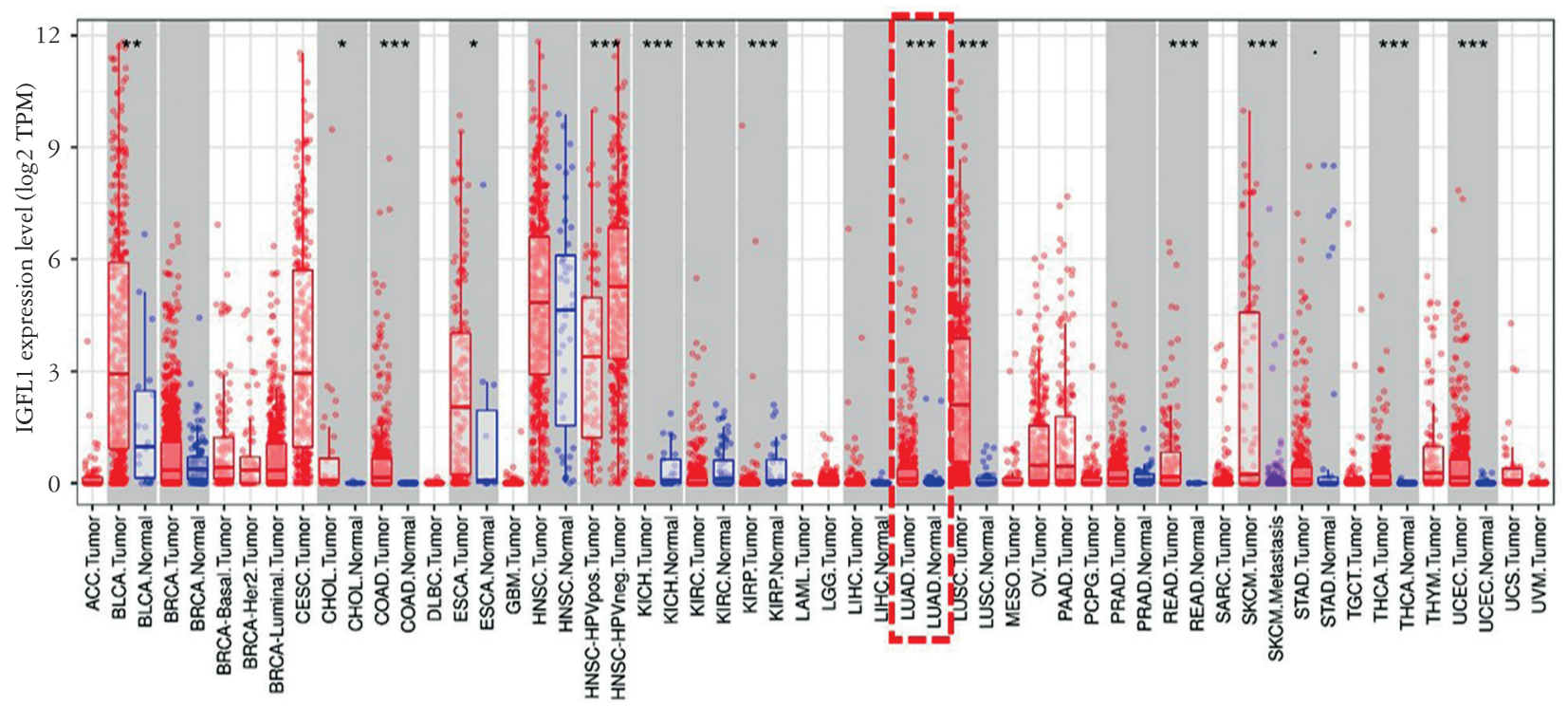

B

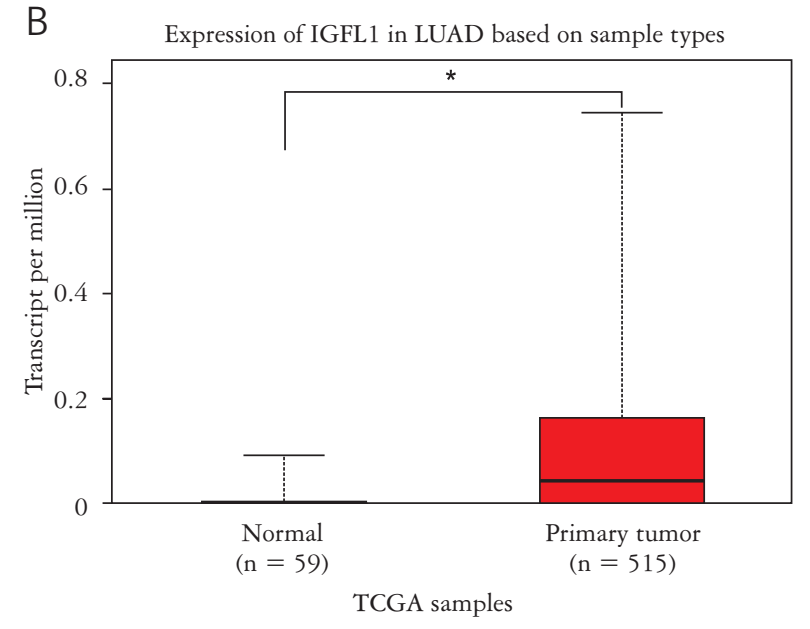

C

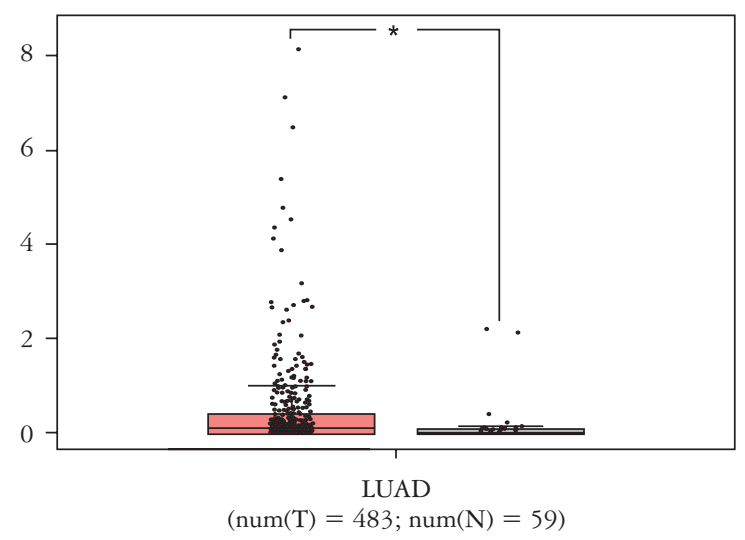

Fig. 1. The expression of IGFL1 in LUAD was predicted and analyzed through bioinformatics analysis. A) Human IGFL1 expression levels in different type of cancers from TCGA database were determined by TIMER $(* \mathrm{p}<0.05$, ** $\mathrm{p}<0.01$, $* * * \mathrm{p}<0.001)$. B, C) Expression of IGFL1 levels in LUAD and normal lung tissue based on TCGA samples in UALCAN (B) and GEPIA (C) $(* \mathrm{p}<0.05)$

of cells $\left(1 \times 10^{4}\right)$ were seeded into 96-well plates and cultured for the indicated time periods $(1,2,3,4$, and 5 days). At the end of different time points, we added $10 \mu \mathrm{l}$ of CCK-8 solution and incubated the cells for a further $2 \mathrm{~h}$. The absorbance was measured at $450 \mathrm{~nm}$ using a microplate reader. The experiment was repeated at least three times.

\section{Cell apoptosis assay}

The A549 and H1299 cells were grown and infected with a lentivirus carrying either the shIGFL1 or shCtrl. Apoptosis was determined using the Annexin V-APC apoptosis kit (eBioscience) according to the manufacturer's instructions. At the end of treatment, cells were collected by trypsinization, washed, and then incubated with Annexin V-APC and binding buffer in the dark at room temperature for $15 \mathrm{~min}$. Finally, flow cytometry analysis was performed using the BD FACSCalibur (BD Biosciences). This assay was performed in triplicate and repeated at least once.

\section{Statistical analysis}

All statistical analyses were performed using SPSS (version 23.0; SPSS Inc. Chicago, USA). The expression of IGFL1 between LUAD and matched adjacent non-tumor tissues was compared using Student's t-test. The relationship between IGFL1 and clinical parameters was analyzed using Pearson's chisquare test. The survival curve was constructed using the Kaplan-Meier method, and the significance of differences was examined using the log-rank test. 
A

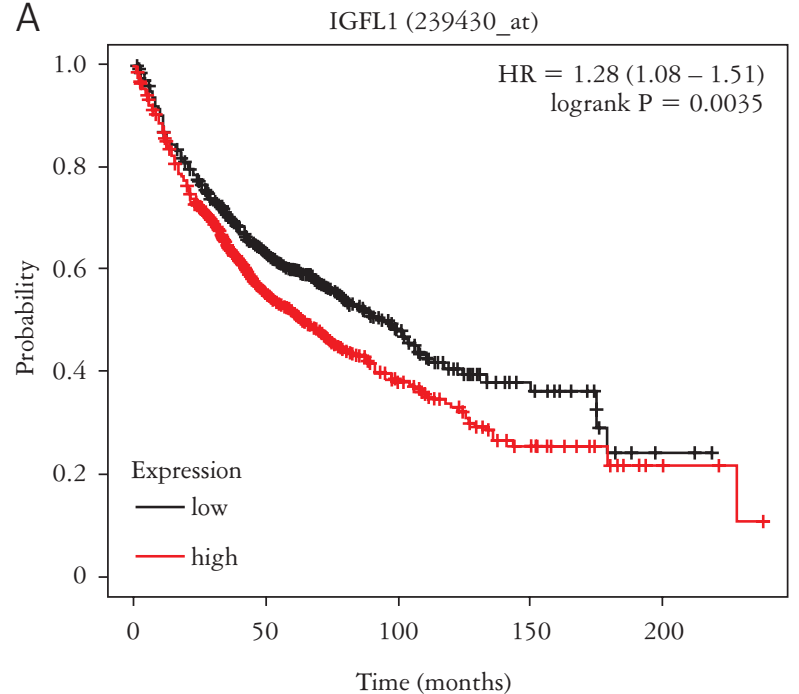

69

$70 \quad 25$
B

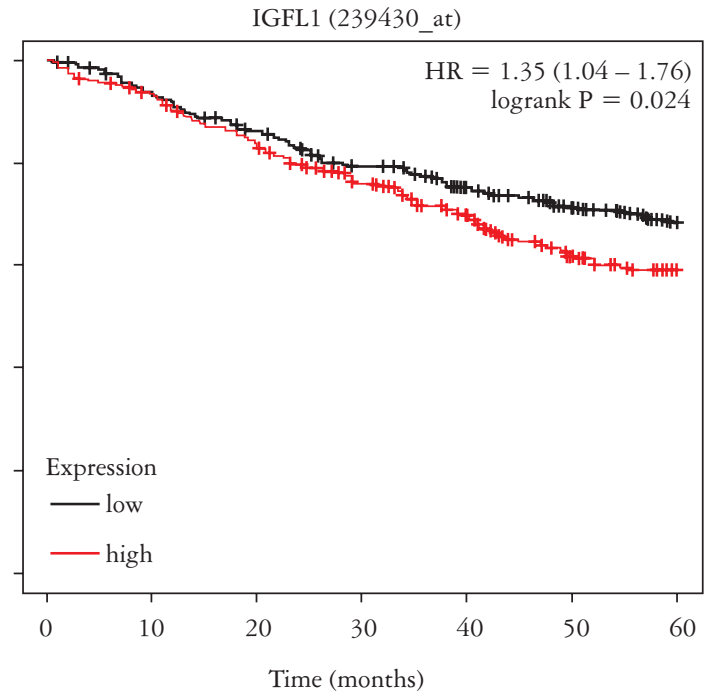

Number at risk

$\begin{array}{rrrrrrrr}\text { low } & 346 & 317 & 287 & 254 & 226 & 200 & 162 \\ \text { high } & 326 & 300 & 266 & 227 & 186 & 144 & 119\end{array}$

Fig. 2. Overexpression of IGFL1 was associated with worse OS in lung cancer (A) and LUAD (B) through Kaplan-Meier plotter (OS - overall survival)

The prognosis of LUAD patients was evaluated using the univariate and multivariate Cox proportional hazards regression models (backward: LR). The hazard ratio (HR) and 95\% confidence interval (CI) were calculated to estimate the hazard risk of the variables. Statistical significance was set at $\mathrm{p}<0.05$.

\section{Results}

IGFL1 is overexpressed in LUAD, as determined using bioinformatics analysis

Tumors are heterogeneous, and the difference in IGFL1 expression in diverse tissues might provide new insights into its function and mechanism. The expression of IGFL1 in different types of cancer was assessed using the TIMER database. This indicated that the expression levels of IGFL1 were significantly increased in bladder urothelial carcinoma (BLCA), cholangiocarcinoma (CHOL), colon adenocarcinoma (COAD), esophageal carcinoma (ESCA), rectum adenocarcinoma (READ), thyroid carcinoma (THCA), uterine corpus endometrial carcinoma (UCEC), LUSC, and LUAD compared to adjacent control samples. However, a decrease was observed in kidney chromophobe ( $\mathrm{KICH})$, kidney renal clear cell carcinoma (KIRC), and kidney renal papillary cell carcinoma (KIRP) (Fig. 1A).

To further confirm the mRNA expression level of IGFL1 in LUAD, UALCAN and GEPIA databases were used. The expression levels of IGFL1 were significantly higher in LUAD samples than in nor- mal lung samples in UALCAN ( $\mathrm{p}<0.05$ ) (Fig. 1B). Meanwhile, in the GEPIA database, IGFL1 mRNA expression levels were also significantly increased in LUAD samples compared to normal lung samples $\left(\log _{2} \mathrm{FC}=0.1, \mathrm{p}<0.05\right)$ (Fig. 1C).

\section{High expression of IGFL1 is associated with poor OS in LUAD}

Furthermore, we investigated the correlation between the overexpression of IGFL1 at the mRNA level and prognosis of lung cancer and LUAD by plotting and comparing the OS using the Kaplan-Meier plotter. The overexpression of IGFL1 was associated with worse OS in lung cancer $(\mathrm{HR}=1.28,95 \%$ CI: $1.08-1.51, \mathrm{p}=0.0035$; Fig. 2A). Moreover, the overexpression of IGFL1 was also associated with poorer OS in LUAD (HR $=1.36,95 \% \mathrm{CI}: 1.04-$ 1.76, $\mathrm{p}=0.024$; Fig. 2B).

\section{Hypo-methylation of the IGFL1 promoter in LUAD}

Abnormal promoter hypomethylation has been reported to induce gene upregulation, which in turn affects tumor progression [21]. In UALCAN, we found that the promoter methylation level of IGFL1 was significantly downregulated in primary LUAD compared with that in normal tissues (Fig. 3A). Patient groups with different gender, TNM stages, and lymph nodal metastasis status showed similar results (Fig. 3B-D). These results suggest that the regula- 
A

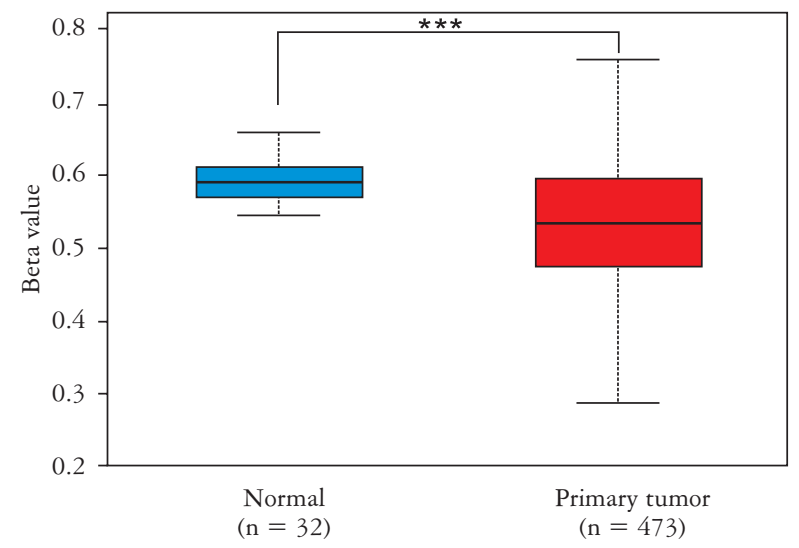

C

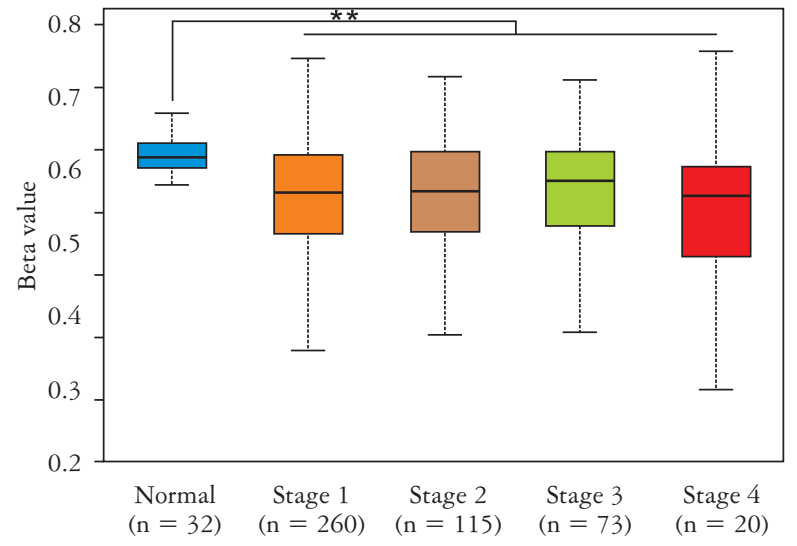

B

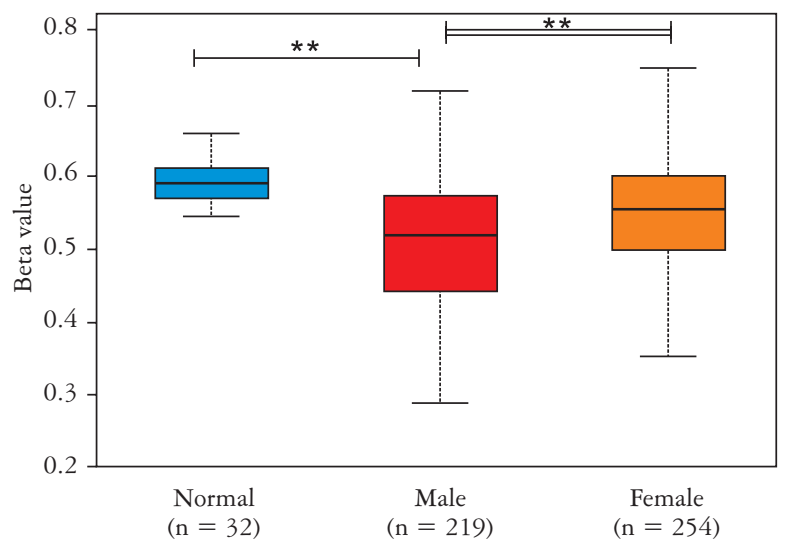

D

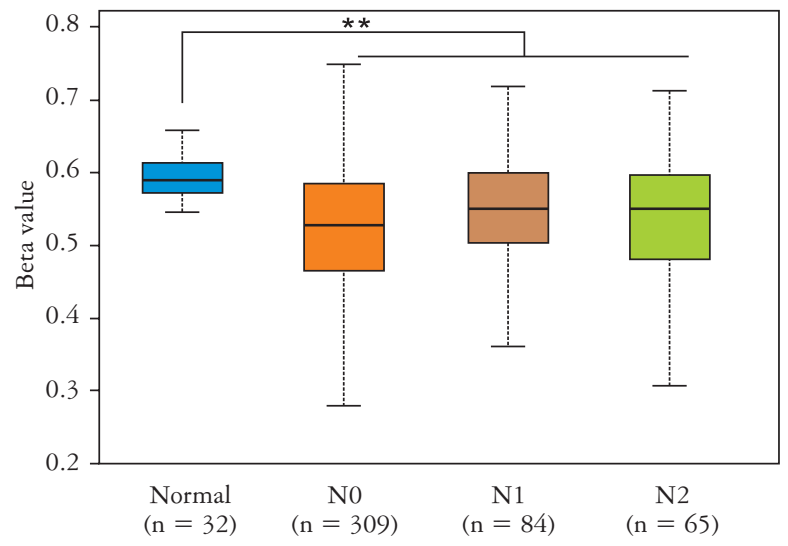

Fig. 3. Analysis of IGFL1 promoter methylation status in LUAD with different clinical parameters in UALCAN. A) The methylation level of IGFL1 promoter in primary LUAD; B) different genders; C) TNM stages; D) LNM status $(* \mathrm{p}<0.05, * * \mathrm{p}<0.01, * * * \mathrm{p}<0.001)$

Table I. IGFL1 is significant overexpressed in LUAD than in adjacent non-tumor tissues.

\begin{tabular}{lccc}
\hline & \multicolumn{2}{c}{ IGFL1 EXPRESSION } & P-VALUE \\
\cline { 2 - 3 } & HIGH & Low & $<0.001^{*}$ \\
\hline Tumor tissue $(\mathrm{n}=208)$ & $149(71.6)$ & $59(28.4)$ & \\
\hline Adjacent non-tumor tissue $(\mathrm{n}=208)$ & $83(39.9)$ & $125(60.1)$ & \\
\hline IGFL1 - insulin growth factor-like family member $1 ; * p<0.05$ was considered significant & &
\end{tabular}

tory mechanism of IGFL1 overexpression in LUAD might be a consequence of promoter de-methylation.

\section{Expression level of IGFL1 is associated with clinical parameters in LUAD patients}

We enrolled patients and divided them into high and low IGFL1 expression groups for further study. We observed that 149 LUAD specimens (71.6\%) had high IGFL1 expression, and $59(28.4 \%)$ had low expression (Table I). The results showed that increased IGFL1 expression in LUAD was significantly associated with pleural invasion $(\mathrm{p}=0.014)$, large tumor size ( $\mathrm{p}=0.010)$, lymph node metastasis $(\mathrm{p}=0.004)$, and high TNM stage $(\mathrm{p}=0.004$; Fig. $5 \mathrm{H}$ ). The relationship between IGFL1 expression and clinical parameters of the patients was assessed (Table II). We then examined the expression of IGFL1 between different histologic subtypes (Fig. 5A-F). Micro-papillary and solid-predominant tumors were more common in the high expression group $(\mathrm{p}=0.008)$. In other words, intermediate 
Table II. Correlation between IGFL1 expression and clinicopathological characteristics of LUAD

\begin{tabular}{|c|c|c|c|c|}
\hline \multirow[t]{2}{*}{ CHARACTERISTICS } & \multirow{2}{*}{$\begin{array}{c}\mathrm{N} \\
(\mathrm{N}=208)\end{array}$} & \multicolumn{2}{|c|}{ IGFL1 EXPRESSION } & \multirow[t]{2}{*}{ P-VALUE } \\
\hline & & $\begin{array}{l}\text { High }(71.6 \%) \\
\quad(\mathrm{N}=149)\end{array}$ & $\begin{array}{c}\text { Low }(28.4 \%) \\
\quad(\mathrm{N}=59)\end{array}$ & \\
\hline Gender & & & & 0.062 \\
\hline Male & 102 & $67(65.7)$ & $35(34.3)$ & \\
\hline Female & 106 & $82(77.4)$ & $24(22.6)$ & \\
\hline Age & & & & 0.057 \\
\hline$\leq 60$ & 92 & $75(81.5)$ & $17(18.5)$ & \\
\hline$>60$ & 106 & $74(69.8)$ & $32(30.2)$ & \\
\hline Smoking history & & & & 0.571 \\
\hline Smoker & 112 & $78(69.6)$ & $34(30.4)$ & \\
\hline Non-smoker & 97 & $71(73.2)$ & $26(26.8)$ & \\
\hline Pleural invasion & & & & $0.014 *$ \\
\hline Yes & 65 & $54(83.1)$ & $11(16.9)$ & \\
\hline No & 144 & $95(66.0)$ & $48(34.0)$ & \\
\hline Tumor size $(\mathrm{cm})$ & & & & $<0.001 *$ \\
\hline$\leq 3$ & 123 & $75(61.0)$ & $48(39.0)$ & \\
\hline$>3$ & 85 & $74(87.1)$ & $11(12.9)$ & \\
\hline Lymph node metastasis & & & & $0.004 *$ \\
\hline Positive & 78 & $65(83.3)$ & $13(16.7)$ & \\
\hline Negative & 130 & $84(64.6)$ & $46(35.4)$ & \\
\hline Pathologic Stage & & & & $0.004 *$ \\
\hline IA & 42 & $21(50.0)$ & $21(50.0)$ & \\
\hline $\mathrm{IB}$ & 43 & $29(67.4)$ & $14(32.6)$ & \\
\hline IIA & 38 & $29(76.3)$ & $9(23.7)$ & \\
\hline IIB & 52 & $42(80.7)$ & $10(19.3)$ & \\
\hline IIIA & 33 & $28(84.5)$ & $5(15.5)$ & \\
\hline LASLC/ATS/ERS a subtype & & & & $0.008 *$ \\
\hline Lepidic & 47 & $28(59.6)$ & $19(40.4)$ & \\
\hline Acinar & 38 & $22(57.9)$ & $16(42.1)$ & \\
\hline Papillary & 40 & $29(72.5)$ & $11(27.5)$ & \\
\hline Micro-papillary & 39 & $32(82.1)$ & $7(17.9)$ & \\
\hline Solid & 44 & $38(86.4)$ & $6(13.6)$ & \\
\hline Architectural grade & & & & $0.001 *$ \\
\hline Low & 47 & $28(59.6)$ & $19(40.4)$ & \\
\hline Intermediate & 78 & $51(65.4)$ & $27(34.6)$ & \\
\hline High & 83 & $70(84.3)$ & $13(15.7)$ & \\
\hline
\end{tabular}

IGFL1 - insulin growth factor-like family member $1 ; * p<0.05$ was considered significant.

and high tumor architectural grades were more common in the group with high IGFL1 expression $(\mathrm{p}=0.001)$.

In 208 clinical LUAD tissues and matched adjacent non-tumor tissues with TMA, the expression of IGFL1 was significantly higher in LUAD tissues than in adjacent non-tumor tissues $(p<0.001$; Fig. 5G). These results confirm the bioinformatic predictions. Consistently, by analyzing the TNM stage of patients, we found that the abnormal expression of IGFL1 was closely correlated with different stages in LUAD patients $(\mathrm{p}=0.004$; Fig. $5 \mathrm{H})$. 


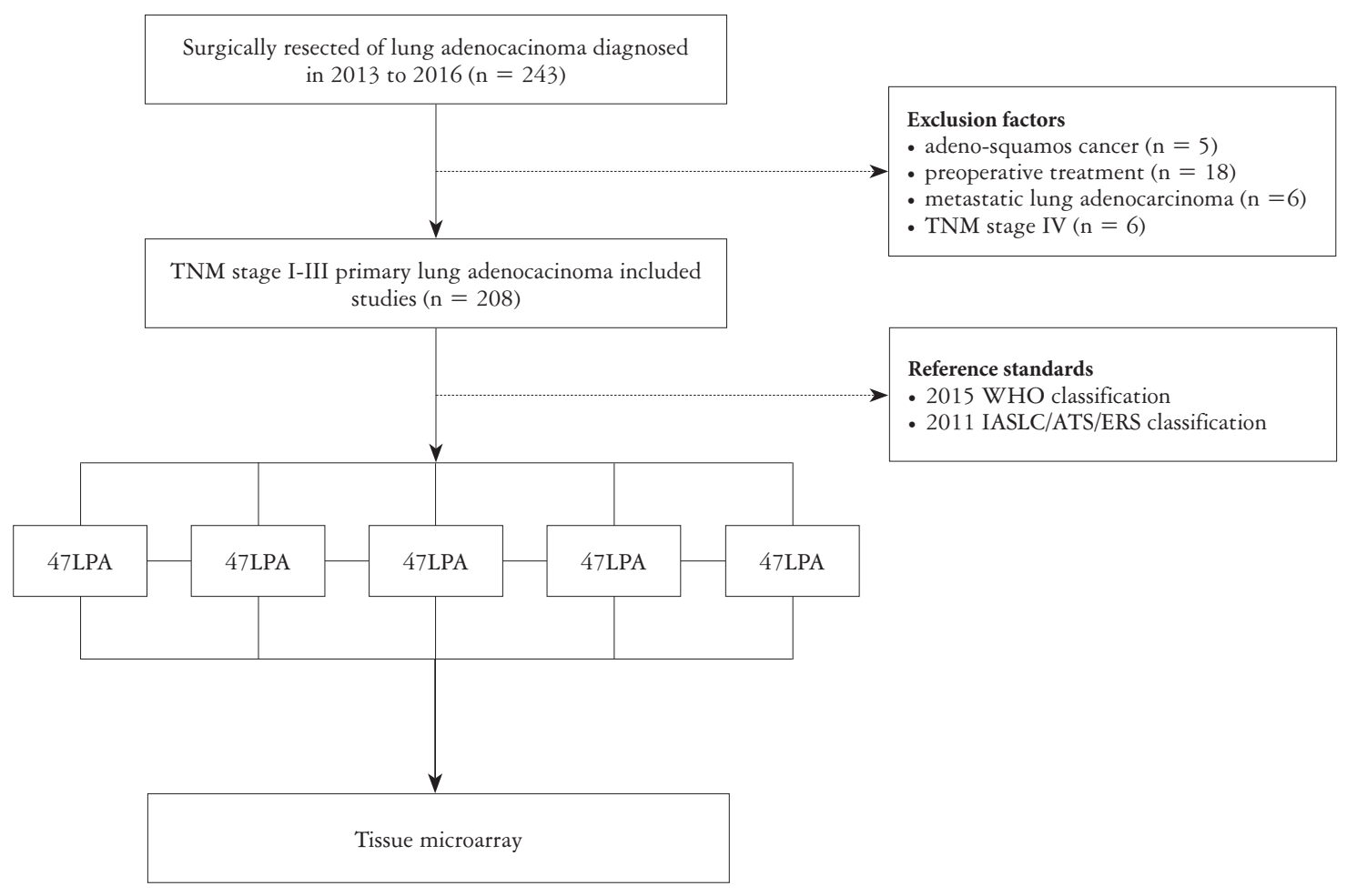

Fig. 4. The screening process for all eligible subjects is shown in the flow chart

\section{The protein level of IGFL1 is associated with OS in LUAD patients in TMA}

Based on the immune reactivity scores of IGFL1 in clinical LUAD samples with TMA, we further verified the correlation between IGFL1 expression and survival in LUAD patients. Furthermore, the Kaplan-Meier survival curve with log-rank test showed that patients with high IGFL1 expression exhibit shorter OS time than those with low IGFL1 expression ( $\mathrm{p}<0.001$; Fig. 4I). To detect the correlation between IGFL1 expression and OS in LUAD patients, univariate Cox's proportional hazard regression was performed, which showed that IGFL1 high expression $(\mathrm{p}<0.001)$, tumor size $>3 \mathrm{~cm}$ $(\mathrm{p}<0.001)$, and higher TNM stage $(\mathrm{p}<0.001)$ were correlated with decreased OS. Subsequently, we further analyzed their independent effects on the OS of LUAD patients using multivariate Cox proportional hazards regression models. The data showed that the expression level of IGFL1 ( $\mathrm{HR}=0.150,95 \% \mathrm{CI}$ : $0.080-0.281 ; \mathrm{p}=0.003$; Table III) could serve as an independent prognostic factor for LUAD patients. In addition, TNM stage II + III $(\mathrm{p}<0.001)$ and tumor size $>3 \mathrm{~cm}(\mathrm{p}=0.007)$ were also found to be independent factors for decreased OS (Table III). Taken together, these data suggest that IGFL1 could function as a biomarker for the prognostic evaluation of LUAD.
IGFL1 enhances the proliferation and inhibits the apoptosis of A549 and H1299 sells

To explore the role of IGFL1 in vitro, we analyzed and tested the expression of IGFL1 in lung cell lines and found that IGFL1 was significantly upregulated in lung cancer cell lines. Based on IGFL1 expression, NSCLC ranked eighth among cancer cell lines in the CCLE analysis (Fig. 6A). We investigated the mRNA levels of IGFL1 in three LUAD cell lines using RT-PCR. Compared with normal lung epithelial cells (BEAS-2B), IGFL1 was more highly expressed in LUAD cell lines (Fig. 6B). Subsequently, we investigated the function of IGFL1 in the growth of LUAD by transfecting A549 and H1299 cells with IGFL1. The RT-PCR assay showed that the mRNA expression of IGFL1 in both cell lines increased after the transfection of the IGFL1 gene compared with that of the control ( $p<0.001$; Fig. 6C). We tested the characteristics of transfected cells to investigate the effects of IGFL1 on tumor progression. The results revealed that knockdown of IGFL1 suppressed the proliferation capacity of A549 and H1299 cells (Fig. 6D-E). The Annexin V-APC assay was used to analyze the effects of IGFL1 on apoptosis of A549 and H1299 cells. Compared with the shCtrl group, the number of apoptotic cells in the shIGFL1 group was increased, indicating that IGFL1 knockdown induced apoptosis in A549 and H1299 cells (Fig. 6F-G). Through a se- 
A

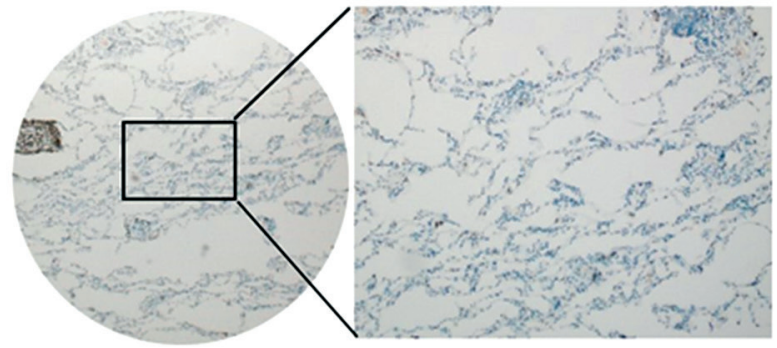

C

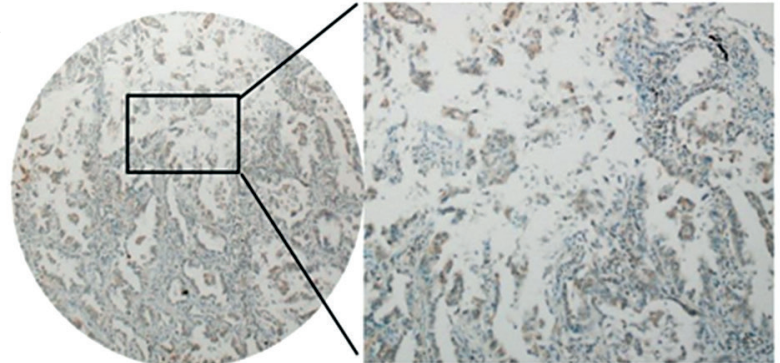

$\mathrm{E}$

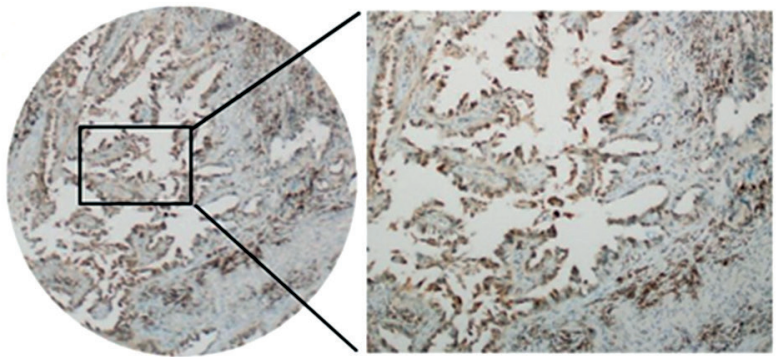

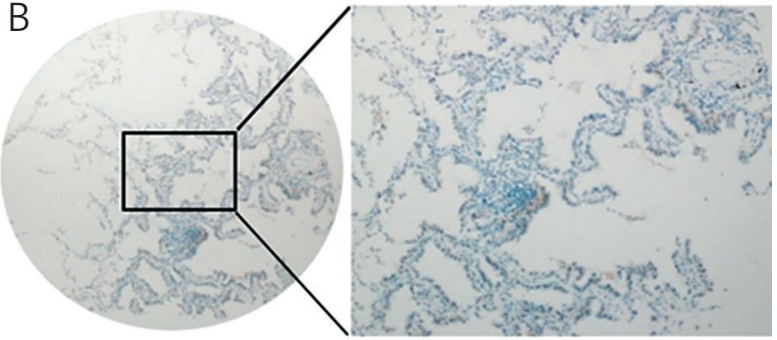
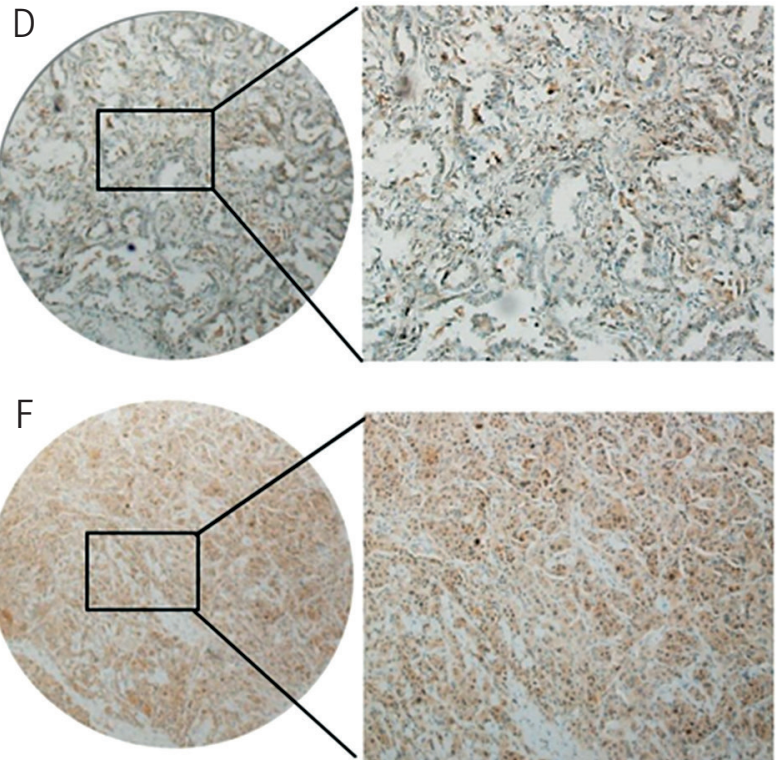

G

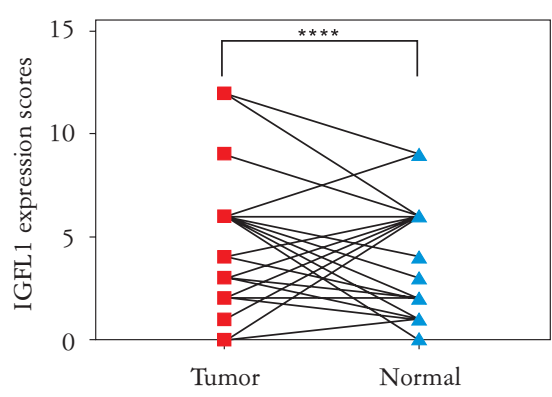

$\mathrm{H}$

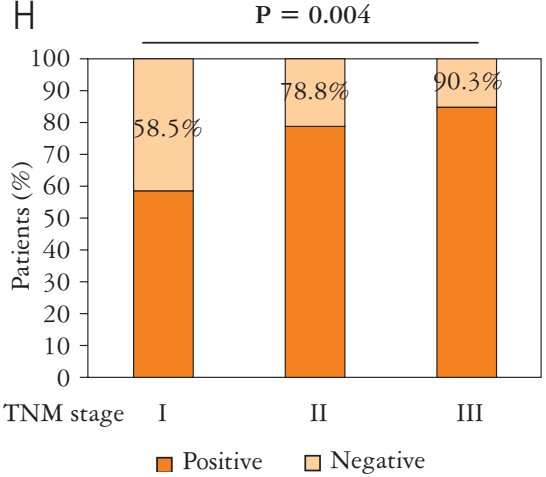

।

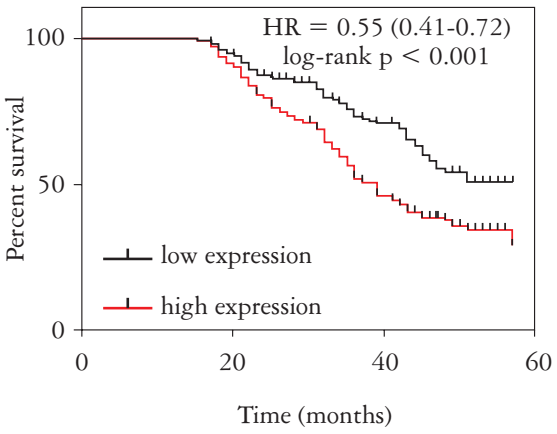

Fig. 5. The expression of IGFL1 in different histological types of LUAD and associated with clinical parameters. A-F) Representative photomicrographs show different expression scores of IGFL1 immunohistochemical staining in LUAD and adjacent non-tumor tissues with TMAs. A) Adjacent non-tumor tissue; negative (0); B) LPA; negative (1+); C) MPA; moderate (2+); D) APA; moderate (2+); E) PPA; strong (3+); F) SPA; strong (3+); magnification: X100 (left); X200 (right). G) Semi-quantitative analysis of IHC results in LUAD and matched adjacent normal lung tissues $(\mathrm{p}<0.001)$. $\mathrm{H})$ Increased percentage of LUAD patients show higher IGFL1 expression in later TNM stages $(\mathrm{p}=0.004)$. I) Higher expression of IGFL1 was associated with worse OS in LUAD $(\mathrm{HR}=0.55[0.41-0.72]$; $\mathrm{p}<0.001)$

ries of experiments, lentiviral-mediated IGFL1 knockdown significantly inhibited the proliferation and tumorigenic ability of LUAD cells, while promoting cell apoptosis.

\section{Discussion}

The incidence of LUAD is increasing annually, and its cancer-related burden is expensive worldwide, which has enormous consequences for society 
Table III. Cox regression analysis of IGFL1 expression and clinical prognostic factors for OS in LUAD

\begin{tabular}{|c|c|c|c|c|c|c|}
\hline \multirow[t]{2}{*}{ FACTORS } & \multicolumn{3}{|c|}{ UNIVARIATE ANALYSIS } & \multicolumn{3}{|c|}{ MULTIVARIATE ANALYSIS } \\
\hline & HR & $95 \% \mathrm{CI}$ & P-VALUE & HR & $95 \% \mathrm{CI}$ & P-VALUE \\
\hline Tumor size $(>3 / \leq 3 \mathrm{~cm})$ & 2.595 & $1.615-4.170$ & $<0.001 *$ & 2.041 & $1.218-3.419$ & $0.007 *$ \\
\hline Lymph node metastasis & 1.107 & $0.678-1.808$ & 0.685 & & & \\
\hline Architectural grade (high + intermediate/low) & 0.745 & $0.541-1.026$ & 0.072 & & & \\
\hline IASLC/ATS/ ERS a subtype & 1.172 & $0.995-1.381$ & 0.058 & & & \\
\hline p-TNM stages $(\mathrm{II}+\mathrm{III} / \mathrm{I})$ & 6.087 & $3.560-10.41$ & $<0.001 *$ & 5.796 & $3.230-10.40$ & $<0.001 *$ \\
\hline IGFL1 (high/low) & 0.293 & $0.137-0.417$ & $<0.001 *$ & 0.150 & $0.080-0.281$ & $<0.001 *$ \\
\hline
\end{tabular}

and economics. LUAD is a highly malignant and heterogeneous disease with various prognoses. Thus, there is an urgent need to identify novel biomarkers to predict patient prognosis and to adjust treatment strategies.

IGFL1 is a novel secreted protein that is rarely expressed in human tissues and has no described biological function. The mature IGFLs are on average shorter than 80 aa and contain 11 cysteines, including two CC motifs. Due to their structural similarity to IGF proteins, IGFLs may also play a similar role in biological processes, such as metabolic control, growth, reproduction, and carcinogenesis[8]. Overexpression of IGFL1 has been previously demonstrated in several types of tumors. IGFL1 exhibits the characteristics of carcinogenesis including hepatocellular carcinoma (HCC) [22], breast cancer [23], and skin malignancy $[11,10]$. Previous studies have demonstrated that IGFL1 levels were increased in stages III-IV in postsurgical HCC tissues, suggesting that IGFL1 is correlated with both the TNM stages and poor prognosis [22]. Moreover, Kirsten et al. reported that the lncRNA IGFL2-AS1 plays a role in down-regulating IGFL1 and affects the migration of breast cancer cells [23]. Studies in mouse models also revealed enhanced expression of mIGFL in skin wounding and psoriatic inflammation [24]. Moreover, due to chronic inflammatory stimuli and immune responses in skin tissues, upregulated IGFL1 may lead to metaplasia [10]. It is known that inflammation plays an important role in cancer from tumor initiation through progression to malignant disease [25]. The above studies all proved that increased expression of IGFL1 is involved in cancer development and progression. In contrast, IGFL1 mRNA was downregulated in co-cultured ESCC cells, compared to the respective mono-cultured ESCC cell lines [12]. This study explored whether IGFL1 may also exhibit the characteristics of a tumor suppressor. Therefore, neither the expression nor prognosis of IGFL1 in LUAD has been clearly established.
In this study, we explored the roles of IGFL1 in LUAD, via an integrated bioinformatic analysis. The cohort from three independent datasets consistently showed that IGFL1 was significantly increased in LUAD tissues compared to normal tissues, indicating that IGFL1 plays a role in tumorigenesis and progression in LUAD. Analysis of IGFL1 promoter methylation status in LUAD with different clinical pathological features in UALCAN demonstrated that the promoter methylation level of IGFL1 was significantly downregulated in primary LUAD compared to that in normal tissues. Patient groups with different gender, TNM stage, and lymph node metastasis status showed similar results. This result explains why IGFL1 abnormal promoter hypomethylation induced gene up-regulation and affected tumor progression.

To investigate the clinical significance of IGFL1 expression at the mRNA level, the relationship between the prognosis of lung cancer and LUAD was analyzed by plotting and comparing the OS using the Kaplan-Meier plotter. We found that IGFL1 mRNA was associated with unfavorable OS in LUAD patients $(p=0.024)$. We analyzed the expression of IGFL1 in LUAD tissues and adjacent normal lung tissues using immunohistochemical tools and evaluated whether high IGFL1 expression levels correlate with the presence of LUAD. Statistical analysis of the expression of IGFL1 proteins revealed a significant relationship with the presence of LUAD $(p<0.05)$. In addition, we investigated the association between IGFL1 expression and other clinical features of LUAD patients. The results revealed that IGFL1 overexpression was closely related to a more advanced pathologic stage ( $\mathrm{p}<0.05)$, special histological subtypes $(\mathrm{p}<0.05)$, and higher architectural grade $(\mathrm{p}<0.05)$, which revealed that IGFL1 may serve as a molecular biomarker for a subpopulation of LUAD patients with a more aggressive disease. In addition, the Kaplan-Meier method and Cox regression analyses showed that IGFL1 could lead to poor prognosis as an independent risk factor. 
A

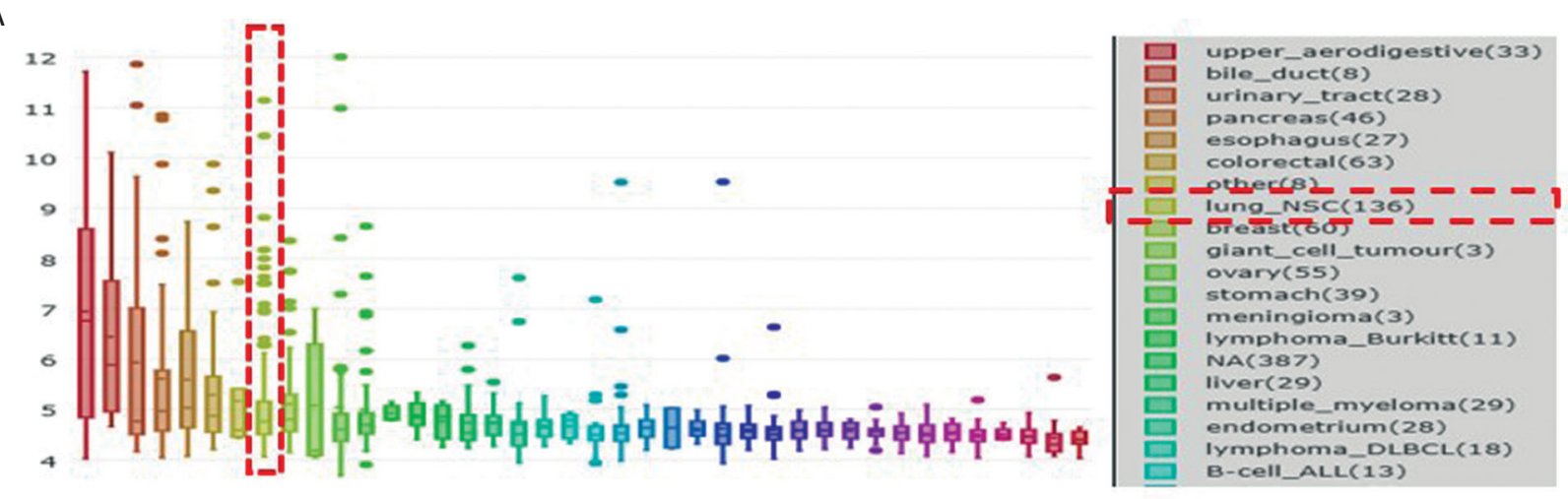

B

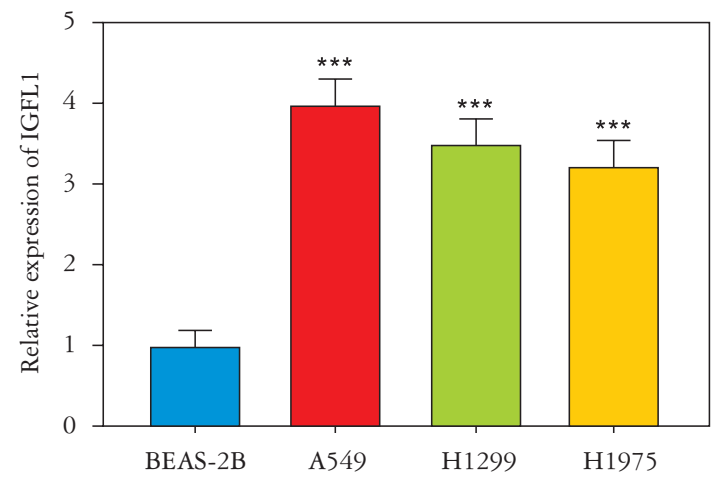

D
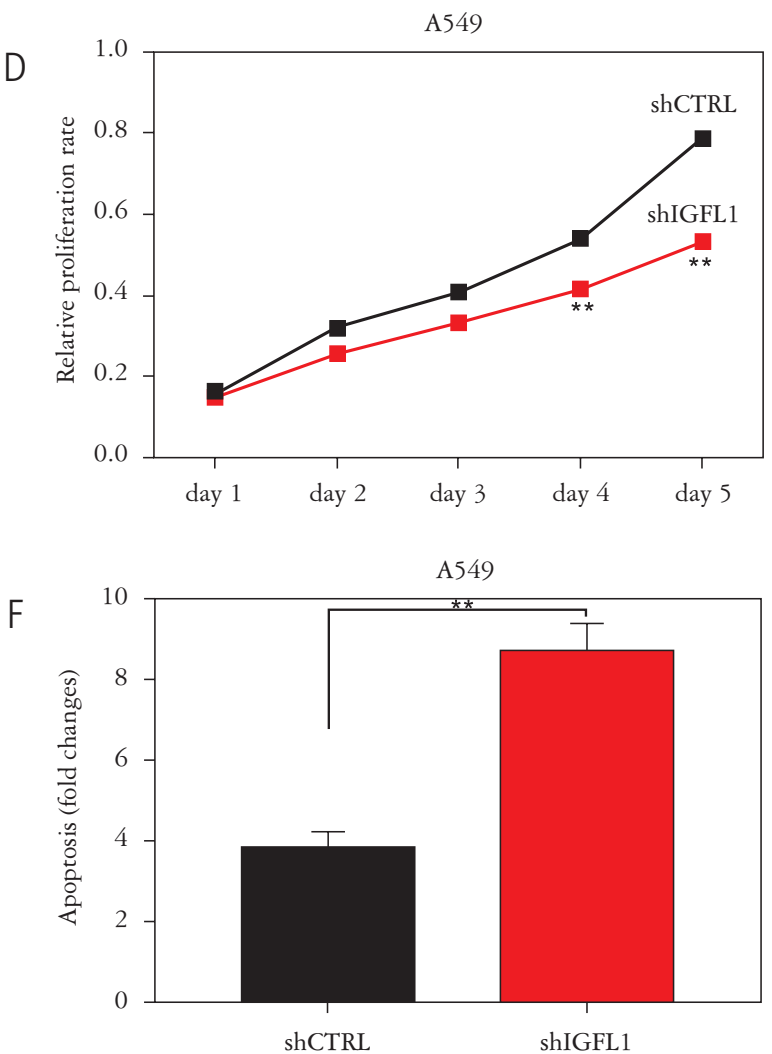

C

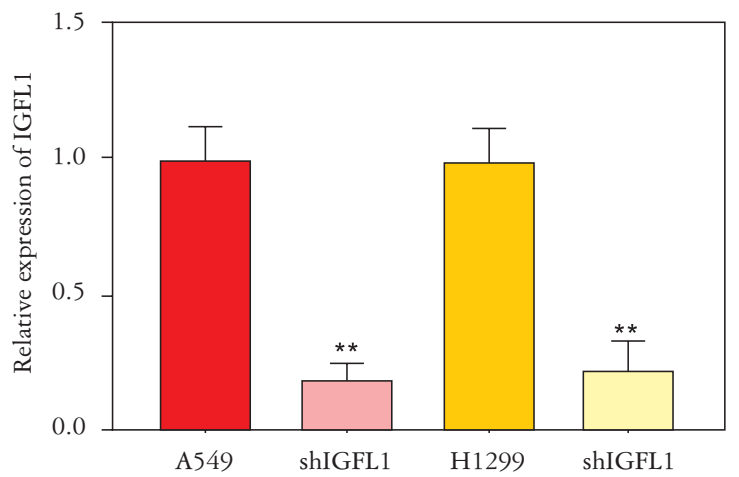

$E$

H1299

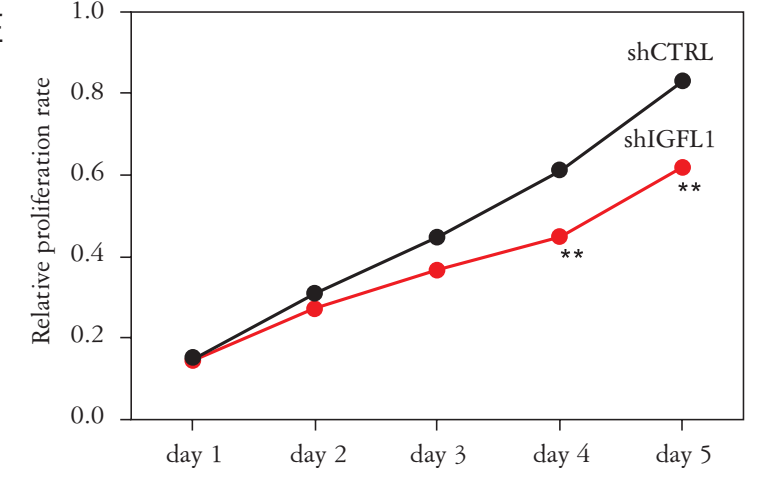

G

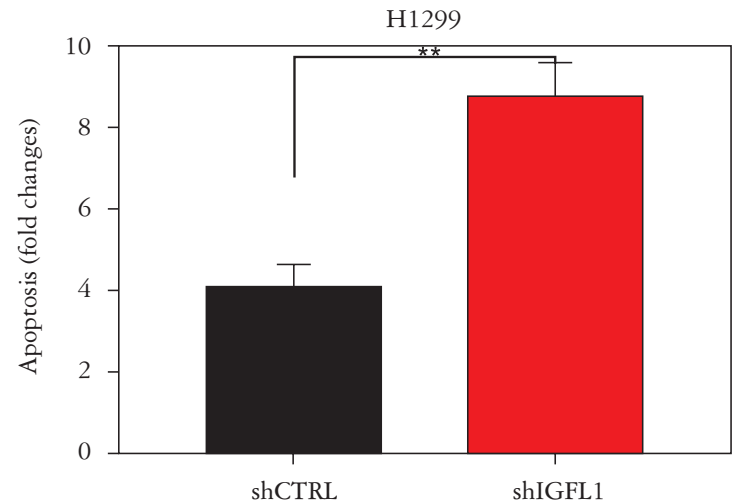

Fig. 6. Functional experiments confirmed that knocking down IGFL1 expression inhibited proliferation and enhanced apoptosis. A) IGFL1 expression in NSCLC cell lines according to data from CCLE database. B) Expression levels of IGFL1 mRNA in A549, H1299, H1975, and BEAS-2B cells by RT-PCR. C) The transfection efficiency of lentivirus-mediated short hairpin RNA was verified by RT-PCR in A549 and H1299 cells. D, E) Cell proliferation ability by CCK8 assay in A5 49 (D) and H1299 (E). F, G) Analysis of apoptosis of A549 (f) and H1299 (g) cells. (* p < 0.05; ** p < 0.01; *** $\mathrm{p}<0.001)$ 
At the cell line level, NSCLC ranked eighth among cancer cell lines based on IGFL1 expression in CCLE. Furthermore, we conducted LUAD cell studies to establish the function of IGFL1 in vitro. Initially, we compared the expression of IGFL1 in three LUAD cell lines and normal lung epithelial cells BEAS-2B, which showed that IGFL1 expression was upregulated in LUAD cell lines, consistent with the results from tissue expression. We further selected the LUAD cell lines A549 and H1299 for subsequent functional studies by inhibiting the expression of IGFL1 via lentiviral-mediated knockdown. Our results revealed that knockdown of IGFL1 significantly inhibited proliferation and promoted apoptosis in A549 and H1299 cells. Taken together, these data suggest that the elevated IGFL1 expression is related to the aggressive clinical parameters of LUAD patients, possibly by promoting proliferation and inhibiting apoptosis. The potential molecular mechanisms involved have not yet been elucidated. In various cell types, with the influence of other hormones or growth factors, IGFLs promote cell proliferation and differentiation and suppress apoptosis [24]. In skin psoriasis, the continuous stimulation of IGFL1 induces the binding of phosphoinositide 3 kinase (PI3K) with IGF-like family receptor 1 (IGFL1R), which triggers downstream protein kinase B (AKT) to promote cell proliferation [26], including promoting DNA synthesis and increasing cell numbers [24]. Activation of the PI3K/AKT pathway plays a critical role in the development of LUAD [27]. Therefore, we suggest that oncogenic IGFL1 transduces downstream signaling through the PI3K/ AKT pathway to promote cell proliferation in LUAD. Further studies that focus on exploring the underlying mechanisms are necessary to identify the potential clinical applications of these findings.

In summary, experimental research was conducted and verified using bioinformatics analysis, many clinical samples, and cellular experiments. These results are the first to demonstrate that IGFL1 is highly expressed in LUAD tissues and is closely related to poor clinical outcomes of patients. It is suggested that IGFL1 may serve as a novel prognostic biomarker. Moreover, we revealed an important role of IGFL1 in promoting proliferation and inhibiting apoptosis in A549 and H1299 cells, which would be helpful for more effective clinical prognosis evaluation. In the future, additional research should be conducted to investigate the effects of IGFL1 proteins on the pathogenesis of LUAD. The identification of potential therapeutic strategies will facilitate the early detection and effective treatment of LUAD patients.

\section{Conclusions}

In the present study, we demonstrated that IGFL1 overexpression frequently occurs in LUAD tissues and is closely related to poor clinical outcomes in patients. We found that IGFL1 can promote tumor cell proliferation and inhibit apoptosis, leading to LUAD progression. This is the first study to reveal the roles of IGFL1 in LUAD, and we suggest that it may serve as a novel prognostic biomarker, which may lead to improving the clinical diagnosis and prognosis evaluation of LUAD. Based on these results, we believe that IGFL1 could be a new therapeutic target in LUAD.

This study was supported by Natural Science Foundation of Gansu Province (Grant No.21JR11RA193) and Science-Technology Foundation for Young Scientists of the Gansu Province of China (Grant No. 18JR3RA059) and the Young Scientists Fund of the Gansu Provincial Hospital of China (Grant No. 2019-306).

The authors declare no conflict of interest.

\section{References}

1. Sung H, Ferlay J, Siegel RL, et al. Global Cancer Statistics 2020: GLOBOCAN Estimates of Incidence and Mortality Worldwide for 36 Cancers in 185 Countries. CA Cancer J Clin 2021; 71: 209-249.

2. Venkatesha VA, Joshi A, Venkataraman M, et al. P7170, a novel inhibitor of mTORC1/mTORC2 and Activin receptor-like Kinase 1 (ALK1) inhibits the growth of non small cell lung cancer. Mol Cancer 2014; 13: 259.

3. Xia L, Zhu Y, Zhang C, et al. Decreased expression of EFCC1 and its prognostic value in lung adenocarcinoma. Ann Transl Med 2019; 7: 672.

4. Varlotto JM, Recht A, Flickinger JC, et al. Factors associated with local and distant recurrence and survival in patients with resected nonsmall cell lung cancer. Cancer 2009; 115: 10591069

5. Cappuzzo F, Tallini G, Finocchiaro G, et al. Insulin-like growth factor receptor 1 (IGF1R) expression and survival in surgically resected non-small-cell lung cancer (NSCLC) patients. Ann Oncol 2010; 21: 562-567.

6. Travis WD, Brambilla E, Riely GJ. New pathologic classification of lung cancer: relevance for clinical practice and clinical trials. J Clin Oncol 2013; 31: 992-1001.

7. Bucciarelli PR, Tan KS, Chudgar NP, et al. BRMS1 Expression in Surgically Resected Lung Adenocarcinoma Predicts Future Metastases and Is Associated with a Poor Prognosis. J Thorac Oncol 2018; 13: 73-84.

8. Emtage P, Vatta P, Arterburn M, et al. IGFL: A secreted family with conserved cysteine residues and similarities to the IGF superfamily. Genomics 2006; 88: 513-520.

9. Khandwala HM, McCutcheon IE, Flyvbjerg A, Friend KE. The effects of insulin-like growth factors on tumorigenesis and neoplastic growth. Endocr Rev 2000; 21: 215-244.

10. Lobito AA, Ramani SR, Tom I, et al. Murine insulin growth factor-like (IGFL) and human IGFL1 proteins are induced in inflammatory skin conditions and bind to a novel tumor necrosis factor receptor family member, IGFLR1. J Biol Chem 2011; 286: 18969-18981.

11. Udensi UK, Cohly HH, Graham-Evans BE, et al. Aberrantly Expressed Genes in HaCaT Keratinocytes Chronically Exposed to Arsenic Trioxide. Biomark Insights 2011; 6: 7-16.

12. Kodaira H, Koma YI, Hosono M, et al. ANXA10 induction by interaction with tumor-associated macrophages promotes 
the growth of esophageal squamous cell carcinoma. Pathol Int 2019; 69: 135-147.

13. Pan JH, Zhou H, Cooper L, et al. LAYN Is a Prognostic Biomarker and Correlated With Immune Infiltrates in Gastric and Colon Cancers. Front Immunol 2019; 10: 6.

14. Chandrashekar DS, Bashel B, Balasubramanya SAH, et al. UALCAN: A Portal for Facilitating Tumor Subgroup Gene Expression and Survival Analyses. Neoplasia 2017; 19: 649-658.

15. Tang Z, Li C, Kang B, Gao G, et al. GEPIA: a web server for cancer and normal gene expression profiling and interactive analyses. Nucleic Acids Res 2017; 45: W98-W102.

16. Györffy B, Lanczky A, Eklund AC, et al. An online survival analysis tool to rapidly assess the effect of 22,277 genes on breast cancer prognosis using microarray data of $1,809 \mathrm{pa}-$ tients. Breast Cancer Res Treat 2010; 123: 725-731.

17. Barretina J, Caponigro G, Stransky N, et al. The Cancer Cell Line Encyclopedia enables predictive modelling of anticancer drug sensitivity. Nature 2012; 483: 603-607.

18. Travis WD, Brambilla E, Nicholson AG, et al.; WHO Panel. The 2015 World Health Organization Classification of Lung Tumors: Impact of Genetic, Clinical and Radiologic Advances Since the 2004 Classification. J Thorac Oncol 2015; 10: 1243 1260.

19. Yoshizawa A, Motoi N, Riely GJ, et al. Impact of proposed IASLC/ATS/ERS classification of lung adenocarcinoma: prognostic subgroups and implications for further revision of staging based on analysis of 514 stage I cases. Mod Pathol 2011; 24: 653-664.

20. Wang X, Xu W, Zhan P, et al. Overexpression of geranylgeranyl diphosphate synthase contributes to tumour metastasis and correlates with poor prognosis of lung adenocarcinoma. J Cell Mol Med 2018; 22: 2177-2189.

21. Van Tongelen A, Loriot A, De Smet C. Oncogenic roles of DNA hypomethylation through the activation of cancer-germline genes. Cancer Lett 2017; 396: 130-137.

22. Zou B, Liu X, Gong Y, et al. A novel 12-marker panel of cancer-associated fibroblasts involved in progression of hepatocellular carcinoma. Cancer Manag Res 2018; 10: 5303-5311.

23. Tracy KM, Tye CE, Page NA, et al. Selective expression of long non-coding RNAs in a breast cancer cell progression model. J Cell Physiol 2018; 233: 1291-1299.

24. Guo P, Luo Y, Mai G, et al. Gene expression profile based classification models of psoriasis. Genomics 2014; 103: 48-55

25. Coussens LM, Werb Z. Inflammation and cancer. Nature 2002; 420: 860-867.

26. Zheng H, Worrall C, Shen H, et al. Selective recruitment of G protein-coupled receptor kinases (GRKs) controls signaling of the insulin-like growth factor 1 receptor. Proc Natl Acad Sci U S A 2012; 109: 7055-7060.

27. Molina-Arcas M, Hancock DC, Sheridan C, et al. Coordinate direct input of both KRAS and IGF1 receptor to activation of PI3 kinase in KRAS-mutant lung cancer. Cancer Discov 2013; 3: 548-563

\section{Address for correspondence}

\section{Qin Yu}

The First Clinical Medical College of Lanzhou University

China

e-mail: yuqin@lzu.edu.cn 